Meta

Journal des traducteurs

Translators' Journal

\title{
Discourse Analysis and the Problem of Translation Equivalence
}

\section{Kazem Lotfipour-Saedi}

Volume 35, numéro 2, juin 1990

URI : https://id.erudit.org/iderudit/003520ar

DOI : https://doi.org/10.7202/003520ar

Aller au sommaire du numéro

Éditeur(s)

Les Presses de l'Université de Montréal

ISSN

0026-0452 (imprimé)

1492-1421 (numérique)

Découvrir la revue

Citer cet article

Lotfipour-Saedi, K. (1990). Discourse Analysis and the Problem of Translation Equivalence. Meta, 35(2), 389-397. https://doi.org/10.7202/003520ar

\section{Résumé de l'article}

The translator's task has usually been defined as the establishment of an equivalence between the source language (SL) and the target language (TL) texts, and the translation process has been characterized as a branch of contrastive linguistics. But neither the nature of translation equivalence (TE) has been carefully specified nor a comprehensive framework consistent with the true nature of linguistic I communicative behaviour has been employed for contrasting languages for translation purposes. Consequently translation studies have always lacked a sound scientific framework.

This paper attempts to study the nature of TE within the framework of a comprehensive contrastive analysis of SL and TL at discourse level and suggests seven different components for TE. 


\section{DISCOURSE ANALYSIS AND THE PROBLEM OF TRANSLATION EQUIVALENCE*}

K. LOTFIPOUR-SAEDI

University of Tabriz, Tabriz, Iran

The translator's task has usually been defined as the establishment of an equivalence between the source language (SL) and the target language (TL) texts, and the translation process has been characterized as a branch of contrastive linguistics. But neither the nature of translation equivalence (TE) has been carefully specified nor a comprehensive framework consistent with the true nature of linguistic/communicative behaviour has been employed for contrasting languages for translation purposes. Consequently translation studies have always lacked a sound scientific framework.

This paper attempts to study the nature of TE within the framework of a comprehensive contrastive analysis of SL and TL at discourse level and suggests seven different components for TE.

\section{1) INTRODUCTION}

Translation process has been defined as a branch of Contrastive Linguistics; and many attempts have been made to characterize the process within the framework of one of the models offered by the modern linguistics for the description of human verbal interaction. But for exactly the same reason that the applied linguists have been disappointed in applying the findings of contrastive linguistics to an effective language pedagogy, the translation studies have failed to define the true nature of the translation process, to characterize the conditions of translation equivalence and to provide a comprehensive framework for determining TE as well as evaluating the translated works. This reason has usually been attributed to the inappropriacy of the linguistic models employed for the purpose. A brief look at the evolutionary trend in the approaches to the study of language would easily reveal the uneasy feeling among the linguists towards this inappropriacy (Hartmann, 1980). One approach has considered the meaning not to be studied in linguistics and has relegated this to such disciplines as philosophy (Cf. Bloomfield, 1933). Others have associated language mainly with syntax and have only focussed on it in their study (Cf. Chomsky et al.). Yet others have brought "meaning" into their descriptive models but the meaning for them has been something inherent in the words and other linguistic elements. In other words, their view of meaning has had a very limited scope. Disillusioned by this limited scope of meaning in linguistics, the investigators have tried to widen the scope and have offered models for describing the "meanings" and "messages" in human verbal interactions in terms of the product of a negotiatory interaction among all the (intra-/extra-linguistic) factors involved in the communication. It is now generally believed that the models employed for studying the human verbal behaviour should be consistent with the true nature of this behaviour, otherwise the studies would be doomed to failure. Translation studies, being concerned with such a behaviour, would not 
be an exception. The linguistic frameworks and models employed for translation studies would thus have to be of such a nature.

Translation is usually defined as the process of establishing equivalence between the source language (SL) and target language (TL) texts. But what are the components of such an equivalence and when is it established? On the basis of the modern trends in discourse analysis and textlinguistics, this paper proposes a comprehensive framework for the characterization of TE. According to this framework, the communicative value of a text (or "an utterance") is the function of the negotiatory interaction of a set of factors and the text can be seen as the surface realization of this interactive discourse process. In other words, all these factors interact with one another for the materialization of the communicative value of a text and the surface text acts as the embodiment of this interactive process. Any change in the surface text and the textual strategies would thus be looked upon as a reflection of a change in the discoursal factors and the change in such factors would obviously be a change in the communicative value or "meaning" of the text. We believe that the problem of TE should be viewed in terms of these discoursal factors and the judgement on whether the TE has been established between an ST text and its alleged translation equivalence should be made in terms of such factors. To put it in other words, TE should be characterized in terms of a negotiatory interaction of all these factors; and these factors can be seen as the integrated conditions of TE. These conditions are as follows: vocabulary, structure, texture, sentence meaning as opposed to utterance meaning, language varieties, aesthetic effect, and cognitive effect. All these seven conditions should be taken into account in the process of establishing TE. We shall elaborate on each of these conditions below; but before doing so it should be emphasized again that these conditions do not act in isolation from one another; but they rather interact with one another for establishing the TE. For example, what may be considered as a TE of an SL vocabulary element in isolation from the other six conditions may turn out to be a completely defective TE in relation to them.

\section{2) VOCABULARY}

The vocabulary of every language can be characterized in terms of a set of interrelated nodes, each node standing for a single vocabulary item. The same as the existence of each node in our network derives mainly from its "location" within the overall network, i.e. from its interrelations with the other nodes in the network, so also does the value of each vocabulary item derive mainly from its relationship with the other vocabulary items within the overall language system, the other intra-linguistic and extra-linguistic factors also being effective in this process. It is the value of the vocabulary items as opposed to their "signification" (Cf. Widdowson, 1978), that should be taken into account in determining the TE of SL vocabulary items.

Every vocabulary item can be seen as consisting of the following layers of meaning and the translator in his attempt to establish the TE should take care of all of these layers.

\section{1) DENOTATIVE MEANING}

Denotative meaning of a vocabulary item derives from the relationship which holds between that item and the realities of the outside world. Not all languages would classify the physical world in the same way (Whorf 1956; Sapir 1949; Saussure 1916). For example, in English the word foot is used to denote the extremity of the leg but in Russian, there is no equivalent for foot. They use noga to denote the whole leg including the foot. Other obvious examples are "kinship" and "colour" terminology in different languages. It is beyond the scope of this paper to discuss the reasons for such difference in 
the lexicalization of the "language substance" or rather "meaning substance" and whether they condition the language user's outlook of the world or not. What we would like to emphasize here is that such differences may cause difficulties in the translation process and so they have to be taken care of.

\section{2) CONNOTATIVE MEANING}

Connotative or experiential meaning is the layer of meaning collected upon the denotative meaning through experience and use. For example, the words "friend" and "comrade" have identical denotative meanings but "comrade" is different from "friend" in terms of its connotation (= used by certain political groups and parties). As another example, we may quote Leech (1974). According to him, the denotative meaning of "woman" can be defined in terms of its meaning components as follows: (+ HUMAN + ADULT + FEMALE). Apart from the denotative meaning, "woman" may have different connotative meanings in different societies and cultures (as an example, "woman in the past has been burdened with such attributes as 'frail', 'prone to tears', 'cowardly', 'emotional', 'irrational', 'inconstant'" p. 14). Connotative meaning of words, due to its nature of source of derivation, is culture-bound; and unlike the denotative meaning, it is indeterminate and open-ended. The culture-specificity of the connotative meaning may be a source of difficulty in the translation process.

\section{3) COLLOCATIVE MEANING}

Part of the meaning of the lexical items, as the structural semanticists would argue, derives from the words or the textual elements with which they co-occur in the text. This aspect of the word meaning is usually referred to as the collocative meaning, which may vary across languages. For example, the Farsi equivalent of the English word "corrupt" in the syntagm: "corrupt ruler" can collocate with nouns like: "eggs", "butter", "fruit", "tooth", etc., whereas in English 'corrupt' cannot co-occur with these and instead, the words "addled", "rancid", "rotten", "decayed" can be used with each one respectively. In determining the TE of SL words, the collocative meaning should also be taken into account.

\section{4) CONTRASTIVE OR PARADIGMATIC MEANING}

Apart from the collocative or syntagmatic meaning, words also have contrastive or paradigmatic relationships with the other words in the lexicon of a language. Contrastive meaning derives from such relationships. In other words, contrastive meaning is the meaning deriving from the relationships which hold among the members of a semantic field. This aspect of word meaning is important in determining the TE of SL vocabulary items, because unless one knows the nature of relationships holding among various items of a lexical field, one is unable to delimit its boundaries and determine its TL equivalence. For example, to determine the Farsi equivalent of the English verb "to poach", the translator should see how it contrasts with the other terms in the English culinary semantic field.

\section{5) STYLISTIC MEANING}

This type of meaning which is also referred to as interpersonal meaning (Halliday 1970 ) is associated with the degree of intimacy between the interlocutors and type of relationship holding between them (necessitating forms such as formal, informal, colloquial, slang, etc.). Two words with equivalent denotative meaning may be of different stylistic meanings. For example: "pass away", "die", "pop off" and "kick the bucket". This aspect of word meaning should also be taken care of in determining the TE for SL vocabulary items. 


\section{6) IMPLICATIVE MEANING}

This refers to the component of the word meaning which is "presupposed" (Kempson 1975), "implied" (Karttunen 1971) or "reflected" (Leech 1974). The TE of SL vocabulary items should never neglect this aspect of meaning. For example the word "territory" (referring to Hong Kong) in the following text:

The Chinese and British Prime Ministers

held talks on the future of the territory.

has specific implicative meaning which should be looked after in the translation process.

\section{3) STRUCTURE}

Structure is the second component of the TE which should be taken into account in the translation process. Three questions arise in this connection: (1) What do we mean by "structure"? (2) Are all languages equal in terms of the number and function of their structural elements? (3) What is the translator's task in translating the structural elements? We shall attempt to provide brief answers to these questions here.

\section{1) WHAT IS MEANT BY THE STRUCTURE?}

Structure deals with the organizational aspects of the elements in a syntagm and consists of compartments such as the following:

a) Features relating to parts of speech, like gender, voice, aspect, mode, person, transitivity, number, time, distance, etc.

b) The order of elements in a syntagm.

c) The concord relationship between the syntagmatic elements.

d) Case-relationships.

e) Direction of the verbal event: who initiates the verbal event and where does it occur...

\section{2) ARE ALL LANGUAGES EQUAL IN TERMS OF THE NUMBER AND FUNCTION} OF STRUCTURAL ELEMENTS?

It is now a principle that the structural resources available to different languages for conveying meanings are not always parallel and identical. For example, not all languages have case-endings to show the case relations; and not all languages possess identical combinations of structural features in their verbal tenses, e.g. "the present perfect" is a separate tense in English but it constitutes a verb category in Russian. Besides, the structural resources and elements which are considered to be identical across languages may vary in terms of their function and communicative value. For example, "passive voice" has been described to have three or four different communicative values in English (see Quirk et al. 1972) one of which is to promote the textual cohesion. To clarify what we mean by this, consider the following two texts:

a) The child ran into the road and was hit by a car.

b) The child ran into the road and a car hit him.

It is now a well-established conception in textlinguistics and cognitive psychology that texts like (a) are more cohesive and thus more easily processable than those like (b). A detailed discussion of the principles lying behind this argument is beyond the scope of this paper (see Lotfipour-Saedi 1986). The language users are thus forced to keep the "thematic elements" of the consecutive sentences in a text identical as far as possible. But it is obvious that not all noun groups have identical case-relations and since the English language has no case-endings to denote the type of case-relations involved, any dislocation of the noun groups in a sentence would disturb their case-relations. Thus to transfer 
the object noun group to the thematic position for the purpose of establishing tighter textual cohesion while keeping its case-relation intact, English utilizes a structure called "passive voice". Persian, due to its special structural features, does not have to use Majhul (which is known to be the Persian counterpart of English Passive Voice) because it possesses a structural device (a kind of preposition) which always accompanies the object noun groups (accusative marker) irrespective of its position in the sentence. Thus passive voice in English and what is considered to be its Persian counterpart do not always perform identical communicative functions.

\section{3) STRUCTURE AND THE TRANSLATOR}

Our discussions in the above sub-sections would suggest that when we include "structure" as a component of TE we do not mean that the translator should replace all the SL structural elements by the TL structures which are considered to be their equivalents without taking their communicative values into account. Such a procedure would obviously lead to structure-for-structure translations which would not always be acceptable and appropriate. What he should rather do is to examine the communicative value of the SL structures and see what TL resources are usually employed for conveying that value.

\section{4) TEXTURE}

Text, as we noted in the introductory section, is the surface realization of the discourse process and embodies a set of strategies for the presentation of the production discourse to the receiver (reader or listener). Text and the textual strategies do not act as the mere "carrier" of the message but actively influence its nature and contents. In other words, the way something is said is as important as the message itself. Textual strategies or texture are thus very important in communication process and should not be neglected by the translator in his attempt to determine TE for SL texts. Among the features relating to texture, we propose the following to be especially taken care of in the translation process:
a) Thematization strategies
b) Textual cohesion
c) Schematic structures
d) Paralinguistic features

Each of these will be further developed upon below.

\section{1) THEMATIZATION STRATEGIES}

Sentence, as a psycho-socio-linguistically determined textual unit plays a crucial role in the linear presentation of the textual strategies (see Lotfipour-Saedi 1982). Every sentence consists of "old" and "new" information units (Halliday 1985) or a "theme" and a "rheme" (Cf. Firbas 1974), and the decision on what should constitute the "theme" and what should be included in the "rheme" of a sentence is made on the basis of certain factors known as the textualization factors (see Lotfipour-Saedi 1982). Any change in the thematic or rhematic components of the sentences of a text would affect the texture and the cognitive processes involved in the discourse comprehension process. The cognitive processes operated by the thematic organization of a text can be argued to be universal and thus any change in such an organization in the translation process would affect the message to be negotiated from the text. The translator should therefore take care of the thematic organization of the SL sentences.

\section{2) TEXTUAL COHESION}

It is obvious that a text (consisting of, say, six sentences) is different from a set of six unrelated sentences. What distinguishes the former from the latter and what constitutes the textness of a text is referred to as textual cohesion (Halliday and Hasan 1976). 
The cohesive elements or the markers of textual cohesion have been classified into "grammatical", "lexical" and "conjunction". It is beyond the limits of this paper to go into the details of these categories. What we should note here is that any careless handling of the cohesive elements of the SL text in the translation process would lead to a change in the degree of cohesiveness intended by the original author and such a change would certainly affect the discourse comprehension processes and in the long run the nature of the message to be negotiated through the text.

\section{3) THE SCHEMATIC STRUCTURE}

Every discourse type or genre is associated with a certain schematic structure. Schematic structures are conventional macro-structures (Cf. van Dijk 1977) which are concerned with the organization of the textual strategies or rather with the rhetorical structure of the speech acts in the speech event. For example, personal letters, commercial and official correspondence, various literary modes (drama, poetry, prose) and reports on psychological experiments each has its own schematic structure. The schematic structure of the SL text should be carefully taken care of in the translation process and despite the fact that there may be differences between the rhetorical structures of various languages (Cf. Houghton and Hoey 1982; Dehghanpisheh 1973), the overall textual layout of the source language and its paragraph organization should be kept intact because any change in such structures and organizations would affect the cognitive processes involved and the textual message in the long run. But certain conventional aspects of the schematic structure of the SL text which are not isomorphic with that of the TL should be replaced in the translation process. For example, personal letters in Farsi mostly have an introductory paragraph of mainly phatic function; and since such introductory paragraphs do not exist in English personal letters, the translator can omit them in translating Farsi personal letters into English.

\section{4) PARALINGUISTIC FEATURES}

Any text, apart from the segmental elements, would also include certain suprasegmental elements which would certainly affect its overall message. These are usually referred to as paralinguistic features which would vary in their realization according to the textual mode. In spoken text, they may realize as intonation, stress patterns, etc., and in written text as underlining, punctuation, italicization, capitalization, bold-type letters, use of brackets and parenthetical sentences, etc. All these features in SL text and their communicative functions intended by the original author should be carefully taken into consideration in determining the translation equivalences.

\section{5) SENTENCE MEANING VS. UTTERANCE MEANING}

By sentence-meaning we mean the meaning which derives from the linguistic elements contained in the sentence without any consideration of the extra-linguistic factors. By utterance meaning we mean the meaning which materializes through an interaction of the linguistic and extra-linguistic factors. For example, the sentence Children will have separate invitations printed at the bottom of an invitation to a wedding, apart from its stated meaning (sentence meaning) would have an utterance meaning saying very indirectly Please do not bring your children. In some cases the two meanings overlap; in some others, as in the case of the example above, they constitute separate dimensions. Semantics is concerned with the study of the sentence meaning and pragmatics deals with the utterance meaning: a broader scope of the meaning catering to the influence of the extra-linguistic factors in verbal interactions. It is beyond the capacity of this short paper to go deeper into the analysis of pragmatic rules. But the knowledge of such rules and an 
awareness of the way the extralinguistic factors monitor the verbal interactions and the materialization of messages and meanings are essential for both the translators and the translation critics because TE should be equivalent not only in terms of the message but also in terms of the way the message is conveyed, i.e. the degree of indirectness the original author has employed for conveying his message. In other words, the SL and TL should also be equivalent in terms of the nature of relationship between the propositional (sentence) meaning and pragmatic (utterance) meaning.

\section{6) LANGUAGE VARIETIES}

A language is not a homogeneous whole; but numerous varieties may come to be classified under one language. Variations in language can be correlated with different geographical, temporal and social factors. Examples of geographical varieties are American English, Australian English and British English; Old English, Middle English and Elizabethan English are examples of temporal varieties; and upper class and lower class English are examples of social varieties. But not all language varieties are equally significant for the translation process. Besides, it is not really possible to determine the TE of certain varieties; for example, what can be the Farsi equivalent of an Elizabethan English text or how can one reflect a certain geographical dialect of English in its Farsi translation? Of all the language varieties, it is mainly the interpersonal variety (the one which is normally classified under the heading of "style" and which is associated with the type and degree of intimacy of the relationship between the interlocutors) which is of significance in translation and which should be given careful consideration. Interpersonal varieties or stylistic variations are in surface realized as changes in both vocabulary and structure. For example, the difference between the following two structures:

Do you want to see me?

Did you want to see me?

and the difference between these vocabulary elements:

horse/steed

die/pass away

are only a matter of style.

Stylistic variations constitute a part of the wider scope of the meaning and are not be taken as "free variations" having no effect on message and being merely a matter of taste. Any careless handling of such variations in the translation process would, thus, yield defective translation equivalents. But the linguistic resources for conveying meanings are not isomorphic in all languages, e.g., one language may reflect the formal variety in its vocabulary while another may employ special structure for this purpose, the translator should not/cannot look at the surface forms in his attempt to determine the translation equivalents. He should rather examine the type of stylistic or interpersonal meaning (the value) of the SL form and variety and then decide what TL textual forms he can choose for conveying the same meaning/value.

\section{7) COGNITIVE EFFECT}

By cognitive effect of a text we mean the effect it may have on the cognitive processes of its recipient (reader or listener), for example, the degree of its comprehensibility and recallability. It is possible for texts which have "equivalent" communicative loads to vary in terms of their cognitive effect. Writers sometimes employ certain strategies to manipulate their readers' cognitive processes forcing them, for example, to process certain 
elements more deeply, to attend to certain other aspects of the message more consciously and to be able to recall certain elements of the message negotiated in the process of reading more vividly some time after reading. We have examined the cognitive function of the textualization strategies in more detail elsewhere (see Lotfipour-Saedi 1986). Here we only note that any change in the textual structure of a text (texture) would affect its cognitive effect. In the translation process, the translator, in his attempt to establish the TL equivalence for the SL text, should not neglect the fact that TE also means equivalence in terms of the cognitive effect. Any irresponsible handling of the SL texture would lead to unwanted "foregrounding" or "backgrounding" of aspects of the original message.

\section{8) AESTHETIC EFFECT}

By aesthetic effect we mean the dimension of meaning added to a text by the literary patterns employed in it. What distinguishes literature from non-literature can be characterized in terms of a set of phonological (rhyming, poetic meters, alliteration, etc.), structural (structural parallelisms, etc.) and semantic (symbols, metaphors, irony, etc.) patterns (Widdowson 1975) super-imposed upon the linguistic segmental code. These patterns affect the meaning intended by the linguistic code both in quantity and quality; and in so doing, they add a new dimension to the meaning, which is lacking in non-literary texts. Thus the meaning in literary texts is created by the interaction of both the linguistic code and the literary patterns:

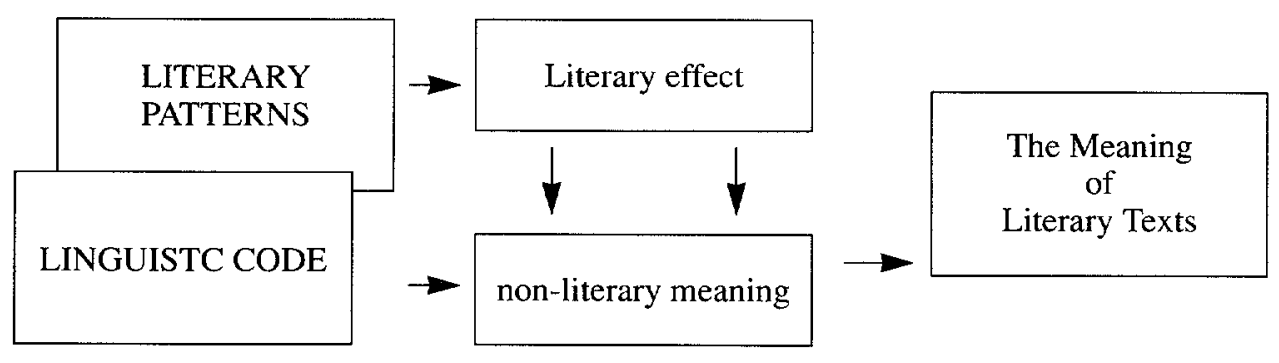

Thus in the translation of literary texts, the translator cannot neglect the SL literary patterns and his TE should be equivalent in terms of both the literary effect and nonliterary meaning. It is beyond the capacity of this paper to go deeper into the problems and issues involved in the translation of literary texts. We have discussed these issues (including the problems of translating metaphors, similes, idioms and expressions, proverbs, jokes, rhyming and alliteration, etc.) in more detail elsewhere (see Lotfipour-Saedi 1984; and forthcoming). The literary patterns, the same as non-literary patterns (i.e. the structure and functioning of the linguistic code), as we noted at the beginning of this paper, may be language-specific and a pattern in one language may either be absent from another one or have a function different from that of the original. So the translator, in his attempt to determine the translation equivalence of the SL literary patterns, should explore their function/value (rather than considering their face/value) first and then try to see what TL literary patterns he can employ for performing the same value. Any careless handling of the SL literary patterns (e.g. translating idiom to non-idiom, metaphor to nonmetaphor or metaphor to simile, etc.) would lead to defective translations. 


\section{9) SUMMARY}

Having defined translation as the process of establishing equivalence between the SL and TL texts, we set out to characterize the nature and components of such an equivalence. We offered the discourse level as a comprehensive framework within which translation studies should be carried out and we chose seven discourse monitoring factors as the conditions for TE. It should once more be emphasized that the nature of a TE should be viewed in terms of all of these conditions interacting with one another but not in isolation from one another.

\section{NOTE}

* Presented to the $8^{\text {th }}$ WORLD CONGRESS OF APPLIED LINGUISTICS, Sydney, 16-21 August, 1987.

\section{BIBLIOGRAPHY}

BLOOMFIELD, L. (1933): Language, George Allen \& Unwin

DEHGHANPISHEH, E. (1973): "Contrastive Analysis of Persian and English Paragraphs" in Proceedings of the Second Annual Seminar of the Association of Professors of English in Iran, Teheran, APEI.

VAN DIJK, T.A. (1977): Text and Context: Explorations in the Semantics and Pragmatics of Discourse, London, Longman.

FIRBAS, JAN (1974): "Some Aspects of the Czechoslovak Approach to Problems of Functional Sentence Perspective" in DANES, F. (ed.): Papers on Functional Sentence Perspective, Prague, Academia Publishing House of the Czechoslovak Academy of Sciences, p. 11-37.

HALLIDAY, M.A.K. (1970): "Language Structure and Language Function", in LYONS, J. (ed.) New Horizons in Linguistics, Penguin, p. 140-165.

HALLIDAY, M.A.K. (1985): An Introduction to Functional Grammar, London, Edward Amold.

HALLIDAY, M.A.K. \& R. HASAN (1976): Cohesion in English, London, Longmans.

HARTMANN, R.R.K. (1980): Contrastive Textology: Comparative Discourse Analysis in Applied Linguistics, Heidelberg, Julius Groos Verlag.

HOUGHTON, Diane \& Michael HOEY (1982): Contrastive Rhetorics, (Unpublished manuscript).

KARTTUNEN, L. (1971): "Implicative Verbs", in Language 47, p. 340-358.

KEMPSON, R. (1975): Presupposition and Delimitation of Semantics, CUP.

LEECH, G.N. (1974): Semantics, Penguin.

LOTFIPOUR-SAEDI, K. (1982): Applying an Analysis of Writer-Reader Discourse Processes to a Pedagogy of Reading, Unpublished Ph.D. Thesis, University of Lancaster, England.

LOTFIPOUR-SAEDI, K. (1984): "Some Issues in the Translation of Literature", paper presented to the First International Conference on Literature in Translation, Javaher-Lal-Nehru University, New-Delhi, March 1984.

LOTFIPOUR-SAEDI, K. (1986): "Textual Strategies and their Cognitive Effect", in English Language Research Journal, University of Birmingham $\mathrm{N}^{\circ} 6$.

LOTFIPOUR-SAEDI, $\mathrm{K}$. (forthcoming): The Theory and Principles of Translation.

QUIRK, R., GREENBAUM, S., LEECH, G.N., and J. SVARTVIK (1972): A Grammar of Contemporary English, London, Longman.

SAPIR, E. (1949): Selected Writings in Language, Culture and Personality (edited by D.G. Mandelbaum), Berkeley, California, University of California Press.

SAUSSURE, F. de (1916): A Course in General Linguistic, New York, Philosophical Library.

WIDDOWSON, H.G. (1975): Stylistics and the Teaching of Literature, London, Longman.

WIDDOWSON, H.G. (1978): Teaching Language as Communication, London, OUP.

WHORF, B.L. (1956): Language, Thought and Reality, Selected Writings, edited by John B. Carroll, The MIT Press. 